\title{
LAS TESIS DEL EMPIRISMO LOGICO Y LA CONVERGENCIA DE LAS DISCIPLINAS
}

Un capítulo de la historia reciente de las relaciones entre las disciplinas ${ }^{1}$

Fernando Salmerón

INSTITUTO DE INVESTIGACIONES Filosóficas UnIVERSidad Nacional Autónoma de MÉxico

\section{Las tesis del empirismo lógico}

Una indagación sobre las disciplinas cientificas y sus relaciones, siquiera sea a grandes trancos y limitada a la etapa que señala el subtítulo general de estas páginas, no puede prescindir del momento en que se encuentran, por primera vez, dos posiciones clásicas: el empirismo lógico y el pragmatismo. Este momento se puede localizar con la aparición del primer volumen de la International Encyclopedia of Unified Science, en el año de 1938.2 Es un momento interesante, porque ofrece la oportunidad de confrontar textos paralelos, en apariencia escritos con una finalidad común, a pesar de los diferentes puntos de partida.

Sobre las finalidades y la estructura de la Enciclopedia, tal como las expone Otto Neurath, hemos dicho algo en otro lugar. ${ }^{3}$ Igualmente sobre las ideas que Rudolf Carnap presenta en su contribución: "Logical Foundations of the Unity of Science", puestas en relación con algunos antecedentes que permitian medir su alcance. Nada de eso es necesario repetir aquí. Sin embargo, conviene intentar una breve recapitulación de las tesis que Carnap defiende en su artículo, con alguna alusión a otras de Reichenbach, con el propósito de conformar una exposición más sistemática. De este modo, se puede hacer más clara la divergencia con el punto de vista de John Dewey sobre las disciplinas científicas y sus rela-

I Estas notas constituyen la segunda lección de un curso sobre "Las disciplinas cientfficas y sus relaciones en la reciente filosofía de la ciencia", dictado en El Colegio Nacional en octubre y noviembre de 1982.

2 International Encyclopedia of Unified Science. Encyclopedia and Unified Science. Volume 1. Number 1. Editor-in-chief Otto Neurath. The University of Chicago Press. Chicago, Illinois, 1938.

En este número colaboran: Otto Neurath, Niels Bohr, John Dewey, Bertrand Russel, Rudolf Carnap y Charles W. Morris.

a Me refiero a un texto titulado: "Sobre el concepto de interdisciplinariedad. Las disciplinas cientificas y sus relaciones en la reciente filosofía de la ciencia", que se encuentra en el libro Lo enseñanza de la fisica. Un enfoque interdisciplinario. SEP, México, 1983. Ambos artículos tienen el mismo origen y el material empleado en ellos fue utilizado en el curso a que se refiere la nota 1. 
ciones. De la misma manera, resultará conveniente redondear la exposición de las tesis de Dewey, con algunas alusiones a ideas de Charles Sanders Peirce, que en tantos aspectos representa su antecedente inmediato.

Hans Reichenbach había tocado el tema de la relación entre la física y las matemáticas, en un pequeño libro sobre los objetivos y métodos del conocimiento físico, en que describía la forma en que trabajan aquellas dos disciplinas como un intercambio permanente o entretejimiento de posibilidad y realidad. ${ }^{*}$ La autonomía de las dos grandes disciplinas es tratada como una distinción que se funda en la naturaleza del conocimiento, dentro de la línea de una tradición filosófica que se remonta a Hume, el verdadero padre del positivismo moderno, según el decir de algún historiador. ${ }^{5}$ De acuerdo a esa distinción, solamente hay dos clases de proposiciones significativas: las que se refieren a cuestiones de hecho y, por tanto, son susceptibles de comprobación empírica; y las de la lógica y las matemáticas, cuya validez es independiente de los objetivos a que se refieren: estas últimas son de suyo evidentes o se infieren de otras proposiciones que sí lo son. De donde deriva la separación entre las ciencias formales y las ciencias empiricas. Como ciencia formal, la lógica es la única posibilidad de fusión de las matemáticas. Pero por el lado de la física, las posibilidades de reducción se extienden, en principio, al dominio de todas las demás ciencias empíricas.

El empirismo consecuente de Reichenbach, le lleva a plantear las cuestiones de la relación entre la física y las demás ciencias empíricas, desde el punto de vista histórico, es decir, desde el punto de vista del desarrollo mismo de cada una de las ciencias. Por eso rechaza cualquier tesis que decida por anticipado el comportamiento de una disciplina en relación a otra. Sus ejemplos, sin embargo, presentados siempre como el resultado final de un desarrollo científico que no hubiera sido posible predecir, abonan las posibilidades de la reducción.

Por otra parte, Reichenbach deja bien claro que la clasificación de las disciplinas empíricas no es algo que pueda plantearse a partir de los objetos de estudio de las ciencias; porque los campos de ciencias diferentes a veces coinciden, a veces interfieren. No se puede trazar una línea divisoria entre las disciplinas, que atraviese también a la naturaleza. El procedimiento indicado debe tomar en cuenta la diversidad de los métodos, que es lo que verdaderamente permite identificar una disciplina. $\mathbf{Y}$

4 EI libro fue publicado en alemán en 1929. La traducción española se imprimió en México en 1945 por el Colegio de México, como primer volumen de una "Colección de Textos clásicos para la Historia de la Ciencia". Hans Reichenbach: Objetivos y métodos del conocimiento fisico. Versión de E. Imaz y prólogo de J. D. Garcla Bacca.

5 Leszek Kolakowski: Positivist Philosophy. From Hume to the Vienna Circle. Trad. de N. Guterman revisada por el autor. Penguin Books, 1972, p. 43. 
una primera comparación descubre que los niveles de madurez y desarrollo de las disciplinas, coinciden con los niveles de exactitud de sus métodos, derivados a su vez de la utilización de conceptos matemáticos. De donde se vuelve nuevamente al asunto de la reducción, porque si el desarrollo de las otras disciplinas empíricas alcanzara alguna vez el punto de madurez de la física - por razón de la exactitud de sus métodos-, resultaría de allí la identidad de los métodos y, en consecuencia, la reducción de tales disciplinas a la física.

De quienes recogieron estos planteamientos nos interesa solamente Carnap, que al introducir el punto de vista lógico modifica la posición de Reichenbach y la convierte en una mucho más radical. Entre los textos de Carnap ha parecido suficiente utilizar un artículo que, por su factura y su línea de argumentación, permite destacar algunas tesis con un máximo de garantía en la fidelidad de la exposición. Se trata de su contribución al primer volumen, ya citado, de la Enciclopedia. Otros textos hubieran podido elegirse, de otros autores o del propio Carnap, pero el señalado permite presentar algunas ideas sobre la ciencia que están en la base de lo que podriamos llamar la versión clásica del empirismo lógico, en cuanto a la manera de considerar las disciplinas científicas y de contemplar el ideal de su unificación. A partir de ellas se pueden establecer ciertas correspondencias con el texto de Dewey, como muestra del punto de vista del pragmatismo.

El tratamiento lógico del problema, tal como Carnap lo plantea, elimina de entrada cualquier consideración sobre la naturaleza de la realidad, que ya Reichenbach había dejado fuera al retrotraer la cuestión a un asunto de mera comparación entre métodos cientificos. Pero además elimina toda discusión de orden histórico acerca del desarrollo de las ciencias, ya no se diga psicológico o social, acerca de las condiciones en que se lleva a cabo la investigación. En el análisis de los enunciados de la ciencia -en general, en todos los enunciados cognoscitivos-, la lógica hace abstracción de las personas y de las condiciones en que se expresan tales enunciados. El problema de la unidad de la ciencia se convierte así en una discusión acerca de la unidad de los términos empleados en cada una de las disciplinas; y de la unidad de las leyes cientificas. Es un problema de lógica, no de ontología.

Otra cosa es que se pueda convenir, para propósitos prácticos, la división del trabajo en la investigación. Pero se tratará siempre de meras convenciones - salvo, tal vez, en el caso de la biología. Frente a la física, que abarca todo el campo no biológico de las ciencias empiricas, es posible establecer una frontera por razones lógico-lingüísticas, en la medida en que los biólogos puedan definir al organismo y a sus procesos como algo irreductible al lenguaje y a las leyes de la física. Pero corresponde 
a ellos la carga de la prueba, y tendrán que mostrar la necesidad de usar otros términos y otras leyes que no pertenezcan a la física, sino que sean específicamente biológicos.

Sin embargo, a los ojos de Carnap, seria cuando menos cuestionable - como no fuera para fines prácticos-, el intento de introducir otras distinciones dentro del amplio campo de la biología: por ejemplo, hablar de ciencias sociales o de psicología, con apoyo en nociones metafisicas de dudosa justificación, como el dualismo mente-cuerpo. Tales intentos tendrian que demostrar la imposibilidad de explicar los comportamientos humanos en tanto que meras conductas de organismos sujetos a leyes biológicas; como tendrían que probar la imposibilidad de tratar con un mismo lenguaje, a los organismos individuales y a los grupos de organismos llamados sociedades. Porque la unidad entre las disciplinas es un asunto exclusivamente lógico y lingüístico. De no existir esa base común, a partir de la cual son posibles los procesos de reducción, no se podrían explicar tampoco las predicciones, fundadas en conocimientos múltiples de varias disciplinas y del sentido común, y en el conjunto de las leyes generales de la ciencia. Y solamente por esas predicciones es posible la aplicación práctica de los conocimientos.

Si ahora intentamos presentar todo esto de una manera más sistemática, sin distinguir la modulación personal que cada filósofo imprime a su punto de vista, ni las rectificaciones menores que, en momentos posteriores, pudo acumular la discusión de los especialistas, es posible resumir aquellas ideas en los seis apartados siguientes. Es posible también, ofrecerlos como las tesis principales del empirismo lógico, a propósito de la unidad de la ciencia y de las relaciones entre las disciplinas.

I) El punto de vista de la lógica de la ciencia. Un enfoque estrictamente lógico y lingǘstico contempla a la ciencia como un cuerpo de conocimientos y, en principio, puede prescindir del contexto social de la investigación, es decir, de cualquier consideración histórica o psicológica acerca de las tareas del investigador.

II) La distinción entre ciencias empiricas y formales es una distinción de conocimientos. La ciencia, como estructura de conceptos, de enunciados y de leyes, combina un tratamiento ampliamente empirista de sus problemas con el auxilio de los instrumentos lógico-matemáticos. Esta distinción, que separa en dos grandes ramas el árbol de la ciencia, se apoya en la naturaleza del conocimiento.

III) En las ciencias empiricas se da una continuidad total. Desde los elementos cognoscitivos del lenguaje ordinario hasta la formulación de las leyes de la física, la ciencia por excelencia, se mantiene aquella continuidad gracias a la unificación de los lenguajes: la clase de los predicados observables de las cosas es suficiente base de reducción para todo 
el conjunto de los lenguajes de la ciencia. Un primer paso se cumple en la reducción de los términos o conceptos de una disciplina a los de otra más fundamental; en un segundo paso, que supone el anterior, se trata de reducción de leyes.

IV) No hay ciencias empiricas diferentes que tengan fuentes de conocimiento diferentes o usen métodos fundamentalmente distintos, sino divisiones convencionales para propósitos prácticos. Hay, sin embargo, en el estado actual de la investigación, un problema lógico aún no resuelto: el de los límites entre las dos grandes disciplinas empíricas, la física y la biología. Muchos procesos biológicos se describen con términos que permanecen irreductibles a los de la física, aunque es un hecho que la investigación se orienta en el sentido de la reducción.

V) El progreso de la ciencia es un avance en los niveles de exactitud pero, sobre todo, de reducción. Los apartados anteriores describen, desde diversos ángulos, una estructura de relaciones lógicas; pero nos dicen poco sobre lo que acontece cuando cambian esas relaciones. Para entender esa estructura en desarrollo tenemos que aceptar -en palabras de Reichenbach-, que el permanente entretejimiento en el trabajo de investigación, de las disciplinas formales y las empíricas, conduce a las segundas a niveles de exactitud, cuyos grados se han de tomar como medidas de progreso. En cuanto a la relación de las disciplinas empíricas entre sí —en esto coinciden Reichenbach y Carnap-, lo que tenemos que aceptar es que el camino de la reducción conduce a la unidad, que es un estadio superior en el desarrollo de la ciencia.

VI) Las leyes cientificas sirven para hacer predicciones; en esto consiste la función práctica de la ciencia. Tomamos decisiones, en la vida individual como en la social, a partir de esas predicciones, que se fundan en conocimiento de hechos concretos de múltiples disciplinas, además de los del sentido común y, por supuesto, de las leyes generales de la ciencia. La participación de esa variedad de conocimientos en las predicciones hace posible la aplicación práctica de todo conocimiento teórico.

El esfuerzo resumido en los seis pasajes numerados no pretendía, por supuesto, la constitución de una disciplina superior de carácter especulativo que, por encima de las ciencias especiales, legislara sobre la forma en que éstas debían cumplir su trabajo. Se presentaba solamente como el análisis descriptivo de una estructura lógica, cuyos rasgos unitarios permitían comprender la forma de operar de la investigación cientifica en su trato con la experiencia y, como consecuencia, la organización de las disciplinas y de sus relaciones. Sin embargo, muchas condiciones contribuyeron a frustrar aquel propósito: entre ellas, la limitación impuesta por el modelo elegido como ciencia ejemplar; la postulación de 
una meta de unidad, que la marcha de la investigación no ha logrado todavía confirmar; y el rechazo de una manera de entender la teoría, en el sentido de contemplación del mundo, que en la tradición filosófica siempre ha implicado la continuidad entre la teoria pura y la práctica vivida.

La ennumeración de estas condiciones no quiere decir, que tales fueran los únicos puntos débiles del conjunto de tesis que hemos resumido. Otros eran menos patentes $o$, tal vez, menos vulnerables dentro de la propia orientación filosófica. Algunos respondían a raices empiristas muy hondas; otros a supervivencias del viejo positivismo; algunos más eran derivados de los avances de la lógica, recientes todavía en aquellas fechas y propensos a favorecer un determinado concepto de racionalidad.

Lo único que no puede decirse, en términos generales, de los defensores de aquellas tesis y de aquel ideal de unidad de la ciencia, es que no estuvieran dispuestos a discutir e incluso a revisar sus opiniones, dentro del espíritu de tolerancia y cooperación intelectual proclamado en la Enciclopedia. Una prueba es el hecho de que en el mismo volumen en que apareció el estudio de Carnap sobre los fundamentos lógicos de la unidad de la ciencia, se publicó otro de Dewey sobre la unidad de la ciencia como problema social, testimonio además del interés de la obra por todos los aspectos de la empresa científica. ${ }^{6}$ De este texto tendremos que ocuparnos ahora, después de señalar algunos antecedentes.

\section{El contexto social de la investigación cientifica}

El ensayo de Dewey no trata de un problema lógico, sino de una cuestión real, es decir, social y cultural acerca de la ciencia. Tal vez, lo primero que tendriamos que advertir, es que no podemos suponer que su autor haya tenido que ocuparse de este problema porque tal fue el asunto que le tocó en el reparto, entre los colaboradores, de los temas de la Enciclopedia. Más bien tenemos que afirmar lo contrario: la obra publicada de Dewey, con anterioridad a este ensayo, como la de sus antecesores y maestros - sobre todo de Peirce-, estaba dirigida justamente a una manera de enfocar el fenómeno de la ciencia, que no era el punto de vista de la lógica formal.

Peirce había iniciado, desde años anteriores al comienzo del siglo, una lucha contra toda forma de subjetivismo. Su experiencia de investigador científico antes que de lógico, le había llevado a tratar de esclarecer el sentido de la ciencia como una actividad, como un proceso autocorrectivo que no tiene comienzo absoluto, ni punto de llegada a salvo

B El título del ensayo de Dewey es "Unity of Science as a Social Problem", pp. 2938 del vol. citado. 
de críticas ulteriores. La convicción de que el conocimiento no se puede legitimar por sus origenes, sino por sus métodos y por las normas de la investigación - aunque estas también son vulnerables a la crítica-, lo llevó a reconocer en todo conocimiento un carácter esencialmente social. En artículos breves, que no tuvieron repercusión hasta que otros autores llamaron la atención sobre ellos, Peirce había propuesto, al lado de estas tesis, una definición de pragmatismo que inició una etapa de la filosofía norteamericana. ${ }^{7} \mathrm{El}$ pragmatismo se presentó ante todo como un método para esclarecer nuestros conceptos, haciendo a un lado confusiones de origen metafísico: era un método para lograr certeza en el significado de las palabras, a partir de la consideración de los efectos de nuestra concepción integral del objeto. Si somos capaces de establecer, siquiera sea en forma hipotética, las consecuencias experimentales de un concepto, entonces sabemos cuál es su significado: cuando dos enunciados producen la misma consecuencia, podemos estar ciertos de que su significado es idéntico.

Durante más de cuarenta años, Dewey había trabajado estas ideas de Peirce y dado a conocer sus desarrollos en libros sobre teoría lógica y en alguno relacionado con problemas de la educación. Pero justo en agosto de 1938 habia firmado el prefacio de su obra mayor sobre estas materias, en la que reclamaba, una vez más, la explicación empírica de las formas lógicas y, a un tiempo, presentaba como innecesaria su interpretación como entidades a priori. ${ }^{8}$ En varios sentidos, la filosofía de Dewey es un esfuerzo por oponerse a los dualismos y pluralismos de la tradición filosófica; un intento de fundar un monismo naturalista sobre la base de no separar de la naturaleza a la experiencia — que es la vida humana en toda su amplitud-, y dentro de la cual se da la investigación como un continuo. Cualquier forma de actividad humana - incluidos conocimientos y valores-, se halla inserta en la naturaleza y tiene un carácter instrumental, es ella misma un instrumento. Singularmente el conocimiento científico. Por eso la palabra verdad no debiera restringirse a una propiedad lógica de las proposiciones, sino extenderse para designar los procesos de la investigación dirigidos de tal manera que realicen un cambio en la naturaleza.

7 Aquí se hace alusión sobre todo a algunos artículos de Peirce: "Questions Concerning certain Faculties Claimed for Man", de 1868; "Grounds of Validity of the Laws of Logic: Further Consequences of Four Incapacities", de 1868; "The Fixation of Belief", de 1877; "How to Make Our Ideas Clear", de 1878; y "What Pragmatism Is", de 1905. Todos recogidos en el Vol. V de Collected Papers of Charles Sanders Peirce, (editado por Ch. Hartshorne y P. Weiss). Harvard University Press, Cambridge, 1960.

8 Se trata del libro fundamental de Dewey: Logic. The Theory of Inquiry, cuya primera edición es de 1938. Hay una edición posterior de Holt, Rinehart and Winston, Nueva York, 1966. 
La ciencia tiene como finalidad el conocimiento, pero el conocimiento no es otra cosa que el conjunto de acontecimientos penetrados por el pensamiento, de tal manera que lo que suele llamarse "ciencia aplicada" puede ser más ciencia en verdad que lo que se llama "ciencia pura", puesto que se ocupa directamente con instrumentos que trabajan para producir modificaciones de la realidad. Así concebido, existe el conocimiento en la ingeniería, la medicina y las artes sociales, más propiamente que en las matemáticas y en la física, que se contentan con fórmulas generales y, por lo mismo, no resultan los instrumentos más poderosos de explicación. ${ }^{9}$ En este sentido, la ciencia reafirma el origen social y práctico del lenguaje, que es especificamente un modo de interacción de al menos dos seres humanos que presuponen la existencia de un objeto: es una comunidad de participación. Las significaciones no son sino reglas para usar y para interpretar las cosas, donde esta interpretación es solamente la hipótesis sobre sus posibles consecuencias experimentales.

En el prefacio de su Lógica. La teoria de la investigación, Dewey advierte la necesidad de desarrollar una teoría general del lenguaje en la que se hallen unidas la materia y la forma, y a partir de la cual se puedan establecer ideas válidas acerca de los conceptos y relaciones que después serán simbolizados. Lo que propone en verdad es una reforma de la lógica, opuesta en todo a las construcciones tradicionales que, según él, se apoyan en teorías psicológicas del conocimiento individual o en el supuesto de que las formas lógicas son formas específicas captadas por la razón. La lógica debe consistir en una investigación del comportamiento real de la investigación científica: las formas y los principios lógicos surgen del proceso de la investigación, y adquieren significado solamente en relación con la situación en que se suscitan, como requisitos para llevar a cabo ese proceso.

Las formas son formas-de-la-materia, y una vez establecidas permiten nuevos modos de operar en la investigación, que a su vez modifican el material del que surgieron. En consecuencia, la distinción entre la manera en que los hombres piensan a veces y aquella otra según la cual deben pensar, no es una distinción entre la psicologia y la lógica. Es

9 Muchas de estas ideas de Dewey se oponen abiertamente a las tesis del empirismo. Su instrumentalismo, por ejemplo, llama la atención sobre la variedad cualitativa de la experiencia, que se entiende en un sentido muy amplio y no da prioridad como criterio de significado a los predicados observables de las cosas. La tesis de la jerarquía cognoscitiva de las disciplinas, en relación inversa a sus niveles de abstracción, contradice al modelo elegido por los empiristas como ciencia ejemplar. Esta última tesis se desarrolla expresamente en otro libro de Dewey: Experience and Nature, cuya segunda edición revisada es de 1929. Hemos utilizado la traducción de José Gaos publicada en 1948 por el Fondo de Cultura Económica. La tesis se encuentra en los párrafos finales del cap. IV, pp. 136 ss. 
simplemente el reconocimiento de dos maneras de investigar: en una época determinada se investiga de acuerdo a ciertos métodos; pero la experiencia puede mostrar, en un momento dado, que son inadecuados y que la tarea debería cumplirse de otra manera. Lo que no significa sino reconocer el carácter tentativo y provisional de todo conocimiento.

El concepto fundamental de la epistemología de Dewey es el concepto de situación, de acuerdo al cual se aclara su concepción práctica y activa del conocimiento. Se trata de una noción intermedia entre dos extremos: el que supone que realidad y conocimiento se construyen a partir de elementos independientes y discretos; y el que pretende que, en último término, realidad y conocimiento son una totalidad simple. Ambos extremos dejan a un lado la realidad del contexto social de la investigación.

Para Dewey, situación quiere decir justo lo contrario de un objeto o un acontecimiento, o una serie de objetos o acontecimientos aislados; porque nunca tenemos experiencia de tales cosas aisladamente, sino siempre dentro de un todo contextual. Y cuando una situación ofrece caracteres confusos, conflictivos o ambiguos - Dewey dice indeterminados-, se dan las condiciones que anteceden a la investigación: porque ésta se define como la transformación dirigida y controlada de una situación indeterminada, en otra cuyas distinciones constituyentes y relaciones quedan convertidas y unificadas en un todo. Dentro de este contexto quedarán incluidas las distinciones que se quiera, como sujeto-objeto, realidad y mundo circundante físico-mental, cantidad y cualidad y muchas otras, en modo alguno previas al proceso de la investigación. El razonamiento no controlado por referencia a una situación determinada, no es razonamiento sino galimatías sin sentido. Un universo de experiencia es la condición previa de un universo de discurso.

Ahora bien, la investigación, como la situación sobre la que actúa, es también un continuo. Desde la determinación de un problema utilizamos los resultados de investigaciones anteriores -y las conclusiones a alcanzar, por seguras que sean, quedan sujetas a rectificaciones posteriores. Los términos del problema son establecidos por la observación, y los intentos de solución son ideas que se ofrecen como hipótesis, para anticipar las consecuencias que habrán de ocurrir si se cumplen ciertas operaciones. La vinculación entre hipótesis y situaciones reales se logra en el examen de la adecuación de esas hipótesis con otras estructuras conceptuales, y en el de su capacidad para recibir la forma necesaria para dirigir un experimento, que permitirá determinar si es aceptable o no. Este tipo de definiciones funcionales elimina cualquier consideración puramente mental de las ideas, que han de enfrentar la prueba de los hechos. Hechos que tampoco son aislados, porque ningún hecho aislado 
tiene capacidad para servir de prueba: esta capacidad deriva de su nivel de organización como elemento de un todo ordenado.

Entre investigación científica y sentido común no se da una diferencia en cuanto a los patrones de la investigación, a sus formas y relaciones básicas. Según Dewey, hay solamente diferencias secundarias que derivan de sus respectivos objetos y finalidades. Hay también diferencias en cuanto a los problemas implicados. Los problemas y las pesquisas del sentido común tienen que ver con las interacciones en que los seres humanos entran en contacto con las condiciones ambientales, para establecer objetos de uso y goce. Los símbolos empleados en tales casos constituyen un sistema eminentemente práctico, formado por tradiciones, técnicas, intereses e instituciones establecidas, que se conectan de manera directa con la vida cultural del grupo. Los significados implicados en este sistema del lenguaje común, determinan la forma en que los individuos, que pertenecen al grupo, pueden usar y gozar de los objetos, de acuerdo a los intereses del propio grupo.

En la ciencia - la cual constituye un lenguaje especializado-, los objetos son estrictamente relacionales, puesto que todos los significados se determinan en razón de su relación recíproca como tales. Las relaciones mismas se convierten en los objetos de la investigación y las cualidades quedan relegadas a un segundo plano - sólo cuentan en la medida en que sirven para el establecimiento de relaciones. En el mundo del sentido común son, en cambio, de importancia definitiva. Todavía la ciencia griega utilizó cualidades como principios explicativos de fenómenos físicos, pero la moderna ha podido dar cuenta de esos fenómenos en términos de relaciones. $\mathrm{Y}$ como estas relaciones son indiferentes a las cualidades, la ciencia puede comportarse con independencia de toda referencia directa a las condiciones y a las preocupaciones del grupo. En esto consiste el carácter abstracto de los objetos científicos que, a pesar de dar lugar a formas secundarias que reflejan el trato con objetos diferentes, no es incompatible con la existencia de patrones o estructuras comunes al comportamiento inquisitivo del sentido común.

La independencia de los objetos científicos de toda referencia limitada y directa al ambiente como factor de actividades de uso y de goce, equivale también a su carácter general, en el sentido de que sus generalizaciones son diferentes de las del sentido común. La generalidad de los objetos cientificos significa que se hallan libres de toda restricción a condiciones que se presentan en tiempos y lugares particulares; pero ésta es una afirmación que no hay que confundir con la teoria de que no mantienen referencia alguna con las condiciones reales. La referencia a tiempo y lugar se halla implicada necesariamente, pero es una referen- 
cia a cualquier serie de existencias que cumplan con las relaciones generales establecidas en la constitución del objeto científico.

\section{Las aplicaciones de la ciencia y la comunidad de los especialistas}

La teoría de la investigación de Dewey hace un recorrido detallado de los grandes temas de la lógica tradicional, y en cada uno de los apartados ofrece el contraste de su enfoque naturalista y pragmático: la cuestión del objeto de la lógica; la construcción de los juicios, términos y proposiciones; la estructura de la investigación; y la lógica del método científico. En el capítulo vi, al comienzo de la segunda parte de su $L \delta$ gica. La teoria de la investigación, se detiene en el análisis de los patrones de la investigación y resume su punto de vista de una manera que hace ver a la tarea como un proceso de descubrimientos, que solamente en segundo lugar lo es de pruebas y modificaciones de hipótesis.

La función conjugada de observación y conceptuación, de hechos y predicciones controladas en tensión recíproca, se traduce invariablemente en operaciones que suponen actividades reales de ideación y órganos de observación, que actúan sobre las condiciones existentes. No se trata de que los logros de un proceso, en tanto que conocimientos teóricos, resulten aptos para tratar la realidad mediante nuevas predicciones que hagan posible su aplicación a situaciones concretas. Se trata de un continuo que no puede distinguir el conocimiento de sus aplicaciones: cualquier operación cognoscitiva tiene una realidad existencial y con su mero planteamiento transforma las condiciones de que partió - más todavła, se trata de que no hay punto de partida. Investigación es igual a transformación; la investigación científica es transformación dirigida y controlada. El objeto de transformación es una situación indeterminada, que deviene unificada y resulta - para ofrecer otra vez nuevas indeterminaciones. Aun los niveles más altos de abstracción, como los objetos de la investigación matemática que han cortado toda referencia a la realidad, son materia de transformación, aunque sea puramente ideal o posible. En tanto que las más concretas aplicaciones de la vida real, no son otra cosa que nuevas formas de corroboración.

Resulta innecesario añadir que Dewey no tendrá reserva alguna frente al ideal de la unidad de la ciencia defendido por los otros colaboradores de la Enciclopedia, en tanto que ideal, pero es bien claro que sus razones son diferentes: no tienen un fundamento lógico en el sentido de Carnap, ni avanzan por el camino de la reducción de las disciplinas. El capitulo dedicado a la investigación social, en Lógica. La teoria de la investigación, establece con toda claridad que el objeto de los problemas sociales es existencial y que, en consecuencia, estas ciencias son ramas de la cien- 
cia natural -en un sentido amplio de "natural". Por supuesto, se reconoce el retraso de las ciencias sociales, pero queda dicho que la cuestión de interés no está en averiguar si el objeto de estas investigaciones puede o no convertirse en una ciencia en el mismo sentido que la física, sino en saber si ese objeto es de una índole tal que permita desarrollar métodos que satisfagan las condiciones lógicas de las disciplinas más exigentes.

El examen del asunto aprueba esa posibilidad -aunque puntualice diferencias--, y el paralelismo de las ciencias sociales con la física resulta tan incuestionable en el texto de Dewey que las conclusiones a propósito de aquellas disciplinas vienen a corroborar lo dicho acerca de ésta. En primer lugar, toda investigación tiene lugar dentro de un cierto cauce cultural, que se halla determinado en último término por la naturaleza de las relaciones sociales. Esto vale para todas las disciplinas: incluso el objeto de la física cae dentro de un campo social más amplio, del que dependen en un cierto momento no sólo las técnicas de la investigación, sino los conceptos generales de la cultura que suministran los medios ideacionales con los que se formulan y abordan los problemas. La sociedad misma proporciona un tejido de conceptos y categorías, dentro del cual se desarrolla el pensamiento individual por original que parezca. Todo lo que la física ha podido hacer para alcanzar una relativa independencia, ha sido desarrollar objetos de investigación y técnicas tan especializadas, que la influencia de las condiciones culturales ha venido a ser puramente indirecta. Justo lo que las ciencias sociales no han logrado.

En segundo lugar, el carácter continuo de la experiencia y la continuidad misma de la investigación que se integra con el rasgo de ser una tarea autocorrectiva, obliga a considerar como garantía suficiente de un juicio, un amplio círculo de consecuencias. Un investigador no puede actuar en forma aislada, sino que tiene que apelar a las experiencias de la comunidad de sus colegas para la confirmación y corrección de los resultados de su trabajo. Aunque la concordancia entre las actividades científicas y las consecuencias prácticas que ellas provocan más allá de la comunidad de los especialistas, en un público más amplio, se hallan en un plano diferente, tal concordancia constituye también parte integrante de la prueba completa de las conclusiones de cualquier disciplina, incluída la física. Siempre que las repercusiones públicas tengan alguna significación.

La afirmación que se acaba de hacer acerca del significado de las repercusiones públicas, no parece aclarar suficientemente el alcance de la concordancia que se reclama como prueba completa. Tampoco lo hacen los comentarios que Dewey añade a su texto, en notas a pie de página 
de este mismo capítulo sobre la investigación social. Pero estas notas permiten seguir la lectura del libro no tanto como una lógica enemiga de todo formalismo, sino como una que supone compatible a la lógica formal, con tal que se reconozca la posibilidad del propio intento: el de dar una explicación empírica de las formas lógicas a partir del análisis del comportamiento de la investigación. Dewey distingue en una nota, por ejemplo, entre la marcha de la investigación y el estado de las creencias en un momento dado, es decir, entre el acuerdo que concierna a actividades - que permite por tanto una ponderación objetiva-, y la aceptación intelectual de la serie correspondiente de proposiciones. Y esta distinción le permite añadir que una proposición no gana en validez por el número de personas que la aceptan. ${ }^{10}$

El amplio círculo de las consecuencias de la investigación —que va más allá de la comunidad científica-, y la idea de la jerarquía de las disciplinas que hemos recordado en el apartado anterior, son perfectamente consecuentes con un ideal de unidad que no requiere de la reducción. La relación entre las ciencias naturales y las sociales es, en todo caso, una relación de condicionamiento, en el sentido de que no es posible comprender los fenómenos sociales si no existe antes una previa comprensión de las condiciones físicas y de las leyes de sus interacciones $-\mathrm{y}$, por supuesto, también de las biológicas. Justo por no haberse dado esta condición, se ha presentado con retraso, según Dewey, la investigación sobre el factor propiamente humano.

Pero el verdadero enemigo de Dewey no es tanto la lógica formal como el empirismo. En el capftulo final de su libro, destinado a revisar rápidamente diversas filosofías del conocimiento, dedica unas páginas a lo que llama "el empirismo y el racionalismo tradicionales". ${ }^{11} \mathrm{Y}$ su objeción va directamente al blanco: considera que esas teorías representan sendos casos de exageración selectiva de un objeto, una del perceptivo, la otra del ideacional. Lejos de aceptar que percepción y concepto son solamente funciones conjugadas y cooperantes, que emergen en el proceso de la investigación, aquellas filosofías suponen que se trata de materiales que proceden de fuentes diferentes.

10 Otras notas son simplemente citas, que apoyan lo que se dice con la autoridad de Cornford, Hodgen y la inevitable de Peirce. La de Cornford es una cita indirecta, tomada de un libro de lógica formal que utiliza la visión historicista de aquel autor para apoyar simultáneamente dos ideas: la que reconoce el peso de la cultura en la formación de los conceptos científicos; y la que distingue entre un uso ordinario del lenguaje y un uso cientifico, que pretende unicidad de referencia $-y$ advierte del peligro de confundir la ciencia con los cientificos. La cita de Cornford proviene de Introducción moderna a la lógica, de L. Susan Stebbing, cuya la. edición es de 1930. Se halla en la p. 34 de la trad. española hecha sobre la séptima edición inglesa de 1954. Centro de Estudios Filos6ficos, UNAM, México, 1965.

11 Logic. The Theory of Inquiry, edicion citada, pp. 516 ss. 
Las críticas van dirigidas expresamente a Kant y a Mill, y no parecen estar al tanto de la producción más reciente del empirismo lógico, menos de los puntos de vista ya publicados en esas fechas por Karl R. Popper. A pesar de lo cual hay también algunos pasajes destinados al "positivismo popular", hijo del empirismo de la tradición. A este positivismo acusa Dewey de desarraigar conceptos para los que no puede encontrar un significado o prueba experimental confirmatoria. Lo acusa también de despreciar las ideas generales y las teorías que se proponen algo más que registrar sumarios de hechos comprobados $y$, sobre todo, de ignorar el papel que han desempeñado, en la historia de la ciencia, hipótesis puramente especulativas o metafísicas. En términos generales, afirma Dewey, ninguna hipótesis cientifica fue verificada nunca en la forma en que se presentó originalmente, sino siempre con modificaciones considerables. La justificación de semejantes hipótesis radica en su capacidad de dirigir nuevas series de observación experimental y abrir nuevos cam. pos de investigación.

La lógica entendida como lógica formal, en cambio, representa a los ojos de Dewey una limitación solamente en la medida en que sea tomada como algo definitivo y autosuficiente, es decir, como el modelo final de la lógica. Semejante manera de hipostasiar los poderes del lenguaje, aísla las formas del discurso racional y obstruye el desarrollo de los modos efectivos de la investigación. Es necesario reconocer la necesidad de operaciones existenciales para poder aplicar los significados del lenguaje a la realidad natural, y aceptar que el mero establecer leyes generales y principios, de hecho y de concepto, es una parte integrante, pero no agota la obra de la ciencia. El continuo de la investigación no se detiene ante las proposiciones singulares.

La falta de claridad en estas cuestiones ha hecho surgir el problema tradicional de las relaciones entre la teoría y la práctica. Para Dewey esto es un falso planteamiento: el empleo de las generalizaciones para el tratamiento de casos singulares en la práctica concreta no es algo extracientífico o puramente "práctico", ni justifica la pretendida diferencia lógica entre ciencias puras y aplicadas. Los procedimientos del médico o del ingeniero no difieren lógicamente de los de los hombres de ciencia que establecen generalizaciones; y pretender otra cosa no pasa de ser una arbitrariedad, probablemente originada en conceptos aristotélicos pero reforzada por el prestigio popular de la física. Desecar pantanos para impedir la cría de cierta clase de moscos y así contribuir a eliminar la malaria, puede ser visto como una tarea práctica, pero desde el lado de la ciencia constituye un experimento que confirma una teoría. Según Dewey, no hay base alguna para trazar una línea lógica que divida las operaciones y técnicas empleadas en la investigación de las ciencias na- 
turales, y las mismas operaciones y técnicas empleadas para fines prácticos. Pero además, la aplicación a las condiciones reales es parte constitutiva intrínseca del método científico.

Ya sabemos que lo que se acaba de afirmar vale para todas las ciencias, pero tratándose de las sociales, la referencia intrínseca a la práctica cobra una fuerza todavía mayor. Los problemas sociales no pueden ser arbitrarios, en el sentido de que, a riesgo de convertirse en meras ficciones, tienen que surgir de las condiciones sociales reales, a veces conflictivas. Además, todo lo que estas ciencias pueden observar - con independencia del cuidado puesto en la empresa y de'la exactitud con que se registre-, solamente puede ser entendido en función de esas consecuencias de actividades proyectadas. Las cuestiones surgen, por tanto, de perturbaciones reales; su objeto de estudio viene a ser determinado por las condiciones, siempre cambiantes, que como obstáculos o recursos permiten llegar a una situación resuelta; y tienen que relacionarse a una hipótesis que represente un plan de acción, una política para la solución real de la situación en conflicto. Lo cual es de una importancia fundamental, porque equivale a que cualquier hipótesis sobre una cuestión social debe abarcar, como parte de sí misma, la idea de una asociación organizada entre aquellos que han de ejecutar las operaciones que la hipótesis formula y dirige.

El antiguo ideal de Peirce de una comunidad genuina de investigadores, comprometidos en una actividad continua y dispuestos a revisar críticamente tanto sus propósitos como los resultados de su trabajo, encuentra en Dewey su complemento final a propósito de la educación. En este asunto, al cual Dewey dedicó la porción más conocida de su obra intelectual, no hemos de detenernos, porque el tratamiento del problema de la unidad de la ciencia como una cuestión social, es un ejemplo suficientemente claro por sí mismo para mostrar la línea de continuidad que enlaza una teoría de la investigación con una filosofía de la acción entendida como teoría crítica. Es decir, una filosofía concebida como un método para discernir, a partir de situaciones reales en materia de creencia, conducta y percepción estimativa, entre los bienes dignos de ser perseguidos, sobre la base de las condiciones en que aparecen y de la consideración de sus consecuencias. La manera de enfrentar la tarea incluirá también un intento de transformación progresiva de las institu* ciones $\mathrm{o}$, mejor dicho, de reconstrucción.

\section{La convergencia de las disciplinas}

La presencia de John Dewey en el corazón mismo de la Enciclopedia internacional de la ciencia unificada, órgano de expresión de un movi- 
miento filosófico que había tenido su origen en países europeos, descubre un nuevo ángulo de preocupaciones en torno a la ciencia que no era visible en la tradición del empirismo lógico. Es el ángulo que pone en primer término al proceso real de la investigación como una empresa humana, que se cumple en un contexto social determinado, dentro de un cauce cultural preciso, y que depende del trabajo efectivo de una comunidad de investigadores. Pero además, el origen de las distinciones entre las disciplinas científicas no tiene que ver con la naturaleza del conocimiento, ni las relaciones entre estas disciplinas son de orden lógico. La investigación científica, como la experiencia, es un continuo que no se ha dividido en partes $y$, por tanto, no puede plantear el problema de su unidad sino como problema social. Aunque, de cualquier modo, en vista de la bondad de sus consecuencias, esta unidad debe ser lograda.

$\mathrm{El}$ ensayo de Dewey comienza por establecer ciertas precisiones a propósito de lo que se quiere decir con el término ciencia, cuando se habla de promover su unidad. En primer lugar, distinguir entre ciencia como actitud y como método, por un lado; y ciencia como cuerpo de conocimientos que son, por otro lado, justamente el resultado del ejercicio de aquel método y actitud. La distinción no significa, por tanto, que se trate de realidades separadas: cada una existe solamente en conexión con la otra. Pero es útil, porque marca la prioridad temporal de la actitud y el método, frente a los materiales que recogen los libros, las revistas científicas y las actas de las academias; y señala también, que la actitud se dirige primariamente hacia los objetos y los acontecimientos del mundo - y sólo secundariamente hacia aquellos materiales que ya son asunto cientificamente elaborado.

La claridad con que Dewey presenta la distinción anterior, le permite subrayar en la ciencia el carácter de producto elaborado por un grupo amplio de personas, que trabajan metódicamente con las realidades de todos los días; y le permite también introducir otras precisiones. Por ejemplo, la relativa a la distinción de las ciencias puras frente a las llamadas aplicadas que, desde luego, considera injustificada. Piensa que establecer esta separación es simplemente un olvido de las tareas necesarias para producir los logros de la tecnología. La ciencia no se aplica automáticamente: su aplicación requiere el uso de métodos que en nada se distinguen de los empleados en los laboratorios. Y quienes llevan a cabo este trabajo son los mismos hombres y utilizan la misma clase de razonamientos.

El tema de la actitud científica da ocasión de puntualizar que, igual que el método, tal actitud no queda confinada a las tareas de los hombres de ciencia: se da al nivel del sentido común, en cualquier actividad de la vida diaria, puesto que no es sino una forma especializada de 
aquélla. Lo mismo sucede con la actitud opuesta. Quienes trabajan por rutina, esclavos de sus dogmas y sus prejuicios, de manera que no están dispuestos a modificar su opinión al tropezar con una prueba en contrario, mantienen una actitud no científica en cualquier actividad de la vida. Al contrario de quienes usan su inteligencia y sus sentidos para interrogar y examinar las cosas, y para sacar sus conclusiones basadas solamente en el análisis de pruebas y argumentos. Por otra parte, es perfectamente posible que los profesionales de la investigación científica no sepan llevar sus buenos hábitos intelectuales más allá de su especialidad, al aceptar en otros campos, creencias o premisas no justificadas, que reciben sin examen previo de su entorno social o de la tradición. En consecuencia, no hay manera de identificar a los hombres dedicados a la investigación cientifica solamente por su actitud; ni tampoco de reducir la actitud científica al ámbito de ciertas tareas especializadas.

Vista en su sentido positivo, la actitud científica se define como la voluntad de inquirir, discriminar y sacar conclusiones a partir de pruebas, después de sopesar todas aquellas disponibles. Todo este esfuerzo, con la intención de alcanzar creencias y de someterlas a prueba basándose en hechos observados; pero además, a partir del reconocimiento de que los hechos carecen de significado - salvo en aquellos casos en que son signos de ideas. De donde se desprende, insiste Dewey, que la actitud experimental -más específica todavía-, tiene que reconocer que las ideas siempre son necesarias para tratar con los hechos, y que estas ideas trabajan como hipótesis, destinadas a ser probadas por las consecuencias que producen.

Sin ir más allá en la caracterización de las actitudes, Dewey desprende de lo dicho hasta aquí, una observación sobre los límites de la ciencia, que puede entenderse a la vez como un intento de trazar una línea de separación entre esta actividad y la metafísica. La vida humana es una permanente interacción con el contorno físico y cultural, y las maneras de enfrentar todo aquello que en la realidad descubrimos como dudoso o problemático son las actitudes. Mientras que la actitud científica hunde sus raíces en la realidad y responde a los problemas que surgen de su trato directo con ella, la no científica los esquiva y los encubre con falsos planteamientos. La experiencia muestra que esta forma de evasión desemboca siempre en problemas artificiales, y a menudo conduce a la búsqueda de soluciones que, en realidad, están decididas de antemano. Ahora bien, el derroche de energía que, para el desarrollo de la actitud científica, representa esta búsqueda errada, no es menor ni más inútil que el de la metafísica - y además, coincide con ésta en razón de una causa común. Hay un sentido de la palabra metafísica que alude justamente al tratamiento de problemas que no tienen su origen en la expe- 
riencia y cuyas soluciones, en consecuencia, se buscan también más allá de ella. Es el límite de la experiencia lo que parece constituir la línea de demarcación.

Si ahora volvemos la atención al tema de la unidad de la ciencia, se verá con claridad que nos encontramos en un terreno completamente distinto al transitado por Carnap y los otros colaboradores de la Enciclopedia. Dewey reconoce expresamente que el problema de unificar los resultados de la ciencia como cuerpo de conocimientos en un conjunto sistemático, es un problema real que no debe ser olvidado, pero no es el que a él le concierne. Lo que merece ser impulsado de manera efectiva, de acuerdo a su propio planteamiento, es la actitud científica; lo que tiene que ser unido son los esfuerzos de aquéllos que practican el método científico en sus labores cotidianas y profesionales; lo que tiene que ser vencido son los obstáculos reales a esta actitud y a este método. Obstáculos que provienen de prejuicios y dogmas, de sentimientos racistas y nacionalistas, de intereses de clase y abusos de autoridad, y de otras agencias similares. Fundamentalmente, se trata de un problema social, cuya relación con la educación habrá que establecer, pero que no puede confundirse con el estudio lógico de la estructura formal de la ciencia, ni con los resultados que este estudio pudiera proporcionar para favorecer la organización sistemática de los conocimientos de las disciplinas especializadas.

Dewey tiene clara conciencia de estas diferencias, pero también de la magnitud de la importancia y de la precedencia del problema social sobre el más propiamente técnico o filosófico. Lo primero, nos dice, debiera ser deliberar sobre el lugar y la función de la ciencia en la escena total de la vida social. Pero además, un movimiento en favor de la unidad de la ciencia no debiera requerir, para su aceptación, el sentar por adelantado una declaración de principios, porque no son éstos los caminos cooperativos que sigue la investigación, y porque de tales avances pueden surgir nuevos obstáculos. Y más adelante afirma, sin añadir ninguna nueva razón para defender su tesis: el intento de asegurar la unidad definiendo los términos de todas las ciencias, en los términos de una sola de ellas, está condenado, por anticipado, al fracaso.

Lo esencial, para Dewey, es que el movimiento de unificación sea un movimiento cooperativo, sin otro acuerdo previo que la fe en la actitud científica y en la importancia social y humana de propiciar su expansión. De esta manera, queda asegurado el surgimiento de ideas y puntos de vista que no estén, por anticipado, atados a tesis que no fueran a su vez fruto del mismo proceso de cooperación.

Las dificultades para la unidad tienen diverso origen para Dewey. En primer lugar, el creciente aislamiento de las especialidades, ocasionado 
por el propio desarrollo de los conocimientos; en segundo, provienen de las diferencias de los lenguajes de cada una de las ramas de la ciencia, que hacen difícil una traducción inteligible para un especialista ajeno a la disciplina. Todo lo cual viene a impedir a los investigadores el uso de instrumentos intelectuales extraños a su especialidad, de que podrían disponer en caso de que se diera libremente un franco intercambio. Para vencer estas dificultades, lo que tiene que intentarse es salvar las brechas y tender puentes de una a otra disciplina. A lo que hay que añadir otra metáfora del mismo autor, para conciliar su visión realista de la marcha de la ciencia con el ideal de unidad. En sus propias palabras: la casa que la ciencia debiera construir, tendría que tener muchas moradas.

En vez de procedimientos reductivos, Dewey habla de convergencia: la tarea consiste en enlazar articuladamente las disciplinas; por ejemplo, las ciencias fisicoquímicas con las psicológicas y sociales, utilizando a la biología como intermediario. Y la mejor manera de alcanzar esta convergencia, puede ser justamente traer varias disciplinas juntas en un ataque común a problemas sociales de orden práctico.

La propuesta parece muy precisa: por una parte, investigar el papel de la ciencia en la vida social, no como cuerpo del conocimiento sino como la empresa real de una comunidad de investigadores que participan de una misma actitud; por otra parte, tender puentes entre las disciplinas, que faciliten la traducción de sus lenguajes y el intercambio de procedimientos intelectuales diversos; finalmente, determinar problemas sociales específicos, de orden práctico, que puedan ser atacados conjuntamente por varias disciplinas. El ideal de la unidad de la ciencia es, ante todo, un ideal de cooperación entre investigadores de diversas disciplinas, con fines de orden práctico, no un ideal de exactitud, ni menos de reducción de unas disciplinas a otras más fundamentales.

Imposible confundir la investigación y la propuesta práctica de Dewey sobre la ciencia como fenómeno social y la convergencia de las disciplinas, con las tesis presentadas por Carnap en el mismo volumen de la Enciclopedia, sobre la lógica de la ciencia considerada como una estructura conceptual. $\mathrm{Y}$ en la medida en que se trata de dos tareas distintas, no hay lugar tampoco —en una primera visión de las cosas-, para poner en duda un cierto grado de compatibilidad, no sólo en tanto que proyectos de investigación sino incluso en relación a sus consecuencias al servicio de una causa común. Esta primera visión explicaria la colaboración de los dos autores en la empresa de la Enciclopedia.

Sin embargo, no conviene minimizar las diferencias sino puntualizar su alcance, en primer lugar con relación a la cuestión de fondo acerca de la lógica; en segundo lugar, con relación a la precedencia de las ta- 
reas; y, finalmente, a propósito de una primera consecuencia de esa precedencia, en cuanto a la marcha misma de la investigación científica.

El ensayo de Dewey es solamente como la parte visible del iceberg. Por debajo de lo expuesto mantiene una concepción de la lógica -presentada en los dos apartados anteriores-, que es otra forma de reducción: nada menos que la explicación empírica de las formas lógicas. Independientemente del nivel de radicalismo en que Dewey sitúa esa premisa, que ahora permanece oculta, es en ella donde habría que buscar el fundamento de la precedencia de su propuesta de investigación social. A ella va unida la consideración del proceso todo de la investigación científica y sus aplicaciones, como un continuo, que se apoya a su vez en la continuidad de la experiencia humana: doctrina que da ocasión a otra tesis sobre la naturaleza del conocimiento, opuesta al dualismo que está en el origen del pensamiento moderno. Continuidad de la experiencia, de la investigación y de la práctica, no es igual a unidad de la ciencia - menos todavía si ésta se entiende como la unidad de la estructura lógica y conceptual de sus enunciados. $\mathrm{Y}$ además, implica una diferencia en la manera de interpretar la función práctica de los conocimientos científicos.

La precedencia de la propuesta de Dewey, en modo alguno implica solamente una diferencia en el orden temporal: se trata de una auténtica alternativa. El mismo Dewey señala expresamente que el camino de Carnap está condenado al fracaso y puede constituir un obstáculo para la unidad de la ciencia, en virtud de que sus puntos de vista no han surgido del proceso cooperativo de la investigación. La verdad es que Dewey conoce perfectamente la magnitud de las diferencias lógicas y epistemológicas de las dos concepciones, aunque en el ensayo de la Enciclopedia reduzca sus reparos a decir que el único requisito para la empresa común debiera ser la fe en la actitud científica.

Una primera consecuencia de esa precedencia de la propuesta de Dewey tiene una significación notable para el tema de las disciplinas científicas y sus relaciones. La sociologia de la ciencia tiene un punto de partida en la actitud científica de las personas; transita una vía efectiva de reconstrucción progresiva de las instituciones; busca una forma de colaboración en la comunidad de los investigadores para enfrentar conjuntamente problemas sociales de orden práctico; y, de esta manera, logra el enlace articulado de las disciplinas que se llama convergencia. El punto de partida, la vía seguida y los resultados señalados no pueden estar más alejados de la lógica de Carnap y de sus propósitos reduccionistas. Porque la idea de convergencia supone, al menos en principio, la aceptación de las disciplinas existentes tal como han ido surgiendo en la marcha de la investigación, con todas las consecuencias que esto 
pudiera implicar — desde las diferencias de sus métodos hasta las peculiaridades de sus lenguajes.

Carnap no pretendía que sus análisis sobre el lenguaje de la ciencia tuvieran un carácter normativo para los investigadores de ninguna disciplina, pero cualquiera que los acepte como válidos no podrá menos de utilizar la vía de la reducción como una hipótesis de trabajo. Dewey, en cambio, se desembaraza de cualquier tesis lógica sobre la unidad de los conceptos o leyes de la ciencia, porque no pretende sino una coincidencia de actitud y un esfuerzo para salvar las brechas entre las disciplinas. Se trata de hacer que diferentes ciencias particulares concurran a un mismo fin, es decir, de hacerlas converger en problemas sociales de orden práctico. Ahora bien, quien comparta esta idea no tendrá más remedio que utilizar en sus tareas de investigación una hipótesis de trabajo diferente: la que supone la existencia de ciencias particulares y, en consecuencia, la ausencia de unidad en el sentido preciso de la reducción de leyes y lenguajes.

Lo que pudiera significar la utilización de esas dos diferentes hipótesis de trabajo en los proyectos de investigación, ha dado lugar a otras polémicas en fechas más recientes. Su estudio podría constituir otro capítulo de la historia de las relaciones entre las disciplinas. 